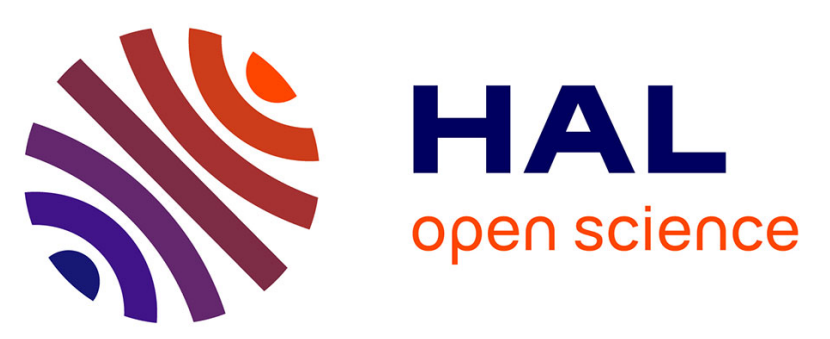

\title{
Structural Models of Poly(cyclopentadithiophene- alt -benzothiadiazole) with Branched Side Chains: Impact of a Single Fluorine Atom on the Crystal Structure and Polymorphism of a Conjugated Polymer
}

Florian Fischer, Navaphun Kayunkid, Daniel Trefz, Sabine Ludwigs, Martin

Brinkmann

\section{To cite this version:}

Florian Fischer, Navaphun Kayunkid, Daniel Trefz, Sabine Ludwigs, Martin Brinkmann. Structural Models of Poly(cyclopentadithiophene- alt -benzothiadiazole) with Branched Side Chains: Impact of a Single Fluorine Atom on the Crystal Structure and Polymorphism of a Conjugated Polymer. Macromolecules, 2015, 48 (12), pp.3974-3982. hal-02045638

\author{
HAL Id: hal-02045638 \\ https://hal.science/hal-02045638
}

Submitted on 4 Jan 2022

HAL is a multi-disciplinary open access archive for the deposit and dissemination of scientific research documents, whether they are published or not. The documents may come from teaching and research institutions in France or abroad, or from public or private research centers.
L'archive ouverte pluridisciplinaire HAL, est destinée au dépôt et à la diffusion de documents scientifiques de niveau recherche, publiés ou non, émanant des établissements d'enseignement et de recherche français ou étrangers, des laboratoires publics ou privés. 
Structural models of poly(cyclopentadithiophene-altbenzothiadiazole) with branched side chains: impact of a single fluorine atom on the crystal structure and polymorphism of a conjugated polymer

Florian S. U. Fischer ${ }^{1}$, Navaphun Kayunkid ${ }^{2,+}$, Daniel Trefz ${ }^{1}$, Sabine Ludwigs ${ }^{1, *}$ and Martin Brinkmann ${ }^{2, *}$

(1) IPOC-Functional Polymers, University of Stuttgart, Pfaffenwaldring 55, 70569 Stuttgart, Germany

(2) Institut Charles Sadron, 23 rue du loess, 67034, Strasbourg, France 
Figure for title of content

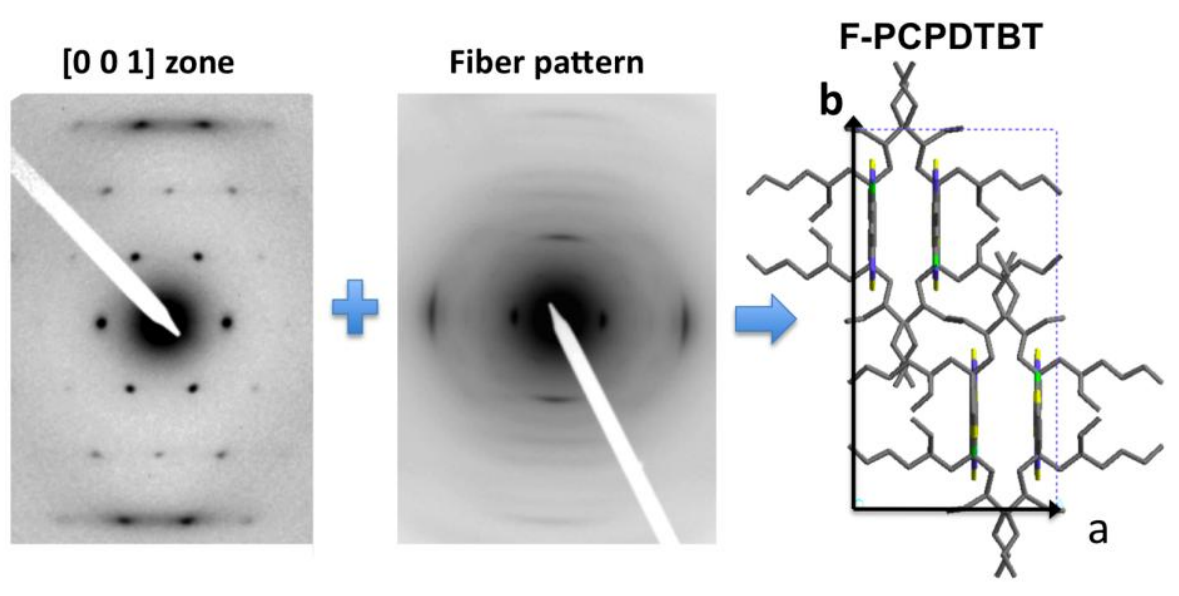




\begin{abstract}
This study describes the impact of a single fluorine atom substitution in the conjugated backbone of poly(cyclopentadithiophene-alt-benzothiadiazole) on the structure and the polymorphism of the polymer bearing 2-ethylhexyl side chains. Controlled growth of the non fluorinated and fluorinated polymers by solvent vapor annealing (SVA), meltcrystallization on oriented poly(tetrafluoroethylene) and high temperature rubbing yield highly crystalline and/or oriented films of a thermodynamically stable polymorph called form I. The single-crystal $h k 0$ and the oriented fiber patterns are obtained in films prepared by SVA and aligned films, respectively. Form I involves a high symmetry packing of four chains in an orthorhombic cell (P c c n space group) with pseudohexagonal symmetry. The chains are paired into dimers with a 3.6-3.8 $\AA$ inter-chain $\pi$ stacking distance and a segregated mode of stacking of cyclopentadithiophene and benzothiadiazole (BT). A single H/F substitution on the BT unit modifies the orientation of the dimers in the unit cell of form I. Absorption and structural properties of this new polymorph are discussed with respect to the classical $\pi$-stacking structure obtained from solvent-additive processed films. Evidence of yet another polymorph (form II) highlights the polymorphism of these alternated donor-acceptor copolymers.
\end{abstract}




\section{Introduction.}

Alternating copolymers made of donor and acceptor units in the backbone have attracted much interest due to the possibility to tune their electronic properties by the adequate combination of donor and acceptor units. ${ }^{1-4}$ This strategy has been particularily fruitful to control opto-electronic properties e.g. the band gap of the active layers in organic photovoltaic cells. From a structural standpoint, the alternation of donor and acceptor units implies different possibilities of $\pi$-stacking between them, the extreme cases corresponding to segregated and to fully mixed stacking. ${ }^{5,6}$ As recently reported for Poly[N,N'-bis(2octyldodecyl)-1,4,5,8-naphthalene-dicarboximide-2,6-diyl]-alt-5,5'-(2,2'-bithiophene)

(P(NDI2OD-T2)) and earlier for Poly(9,9-di-n-octylfluorene-alt-benzothiadiazole), two different polymorphs corresponding to these two stacking modes were identified and the consequences in terms of optical and device properties were uncovered. ${ }^{5-7}$ However, to establish structure-property correlations in such complex macromolecular systems, it is necessary to generate high order and crystallinity, which is challenging. A major breakthrough has been achieved by using solvent vapor annealing that leads to highly crystalline films as illustrated recently for $\pi$-conjugated polymers such as regioregular poly(3hexylthiophene) (P3HT) and Poly[2,6-(4,4-bis-(2-ethylhexyl)-4H-cyclopenta[2,1- $b ; 3,4-$ $b^{\prime}$ ]dithiophene)-alt-4,7(2,1,3-benzothiadiazole)] (PCPDTBT). ${ }^{8,9}$ Other approaches to induce high crystallinity and orientation were proposed such as zone casting ${ }^{10}$, epitaxy ${ }^{11}$ or hightemperature rubbing. ${ }^{12}$

Most semiconducting polymers made of a conjugated backbone bearing alkyl side chains have a "layered" structure made of an alternation of $\pi$-stacked backbones and layers of more or less ordered side chains. ${ }^{13}$ This is the case for major polymers such as regioregular poly(3alkylthiophene)s and poly(2,5-bis(3-dodecyl-2-yl)-thieno[3,2-b]thiophene) (PBTTT). ${ }^{13,14}$ 
However, this type of "smectic-like" ordering is not the only one observed. In the case of the alpha form of poly $\left(9,9^{\prime}\right.$-dioctylfluorene) (PF8) and the gamma form of poly $\left(9,9^{\prime}-\right.$ diheptylfluorene) (PF7), the chains are grouped into dimers that ultimately form an ordered orthorhombic structure with high symmetry ( $\mathrm{P} \mathrm{n} \mathrm{b} 21$ for PF8). ${ }^{15,16}$ In these systems, and contrary to P3HT or PBTTT, no long-range $\pi$-stacking of the chains is observed.

Regarding the structure of PCPDTBT, several studies by Grazing Incidence X-ray Diffraction (GIXD) on marginally crystalline films obtained by spin-coating suggest that PCPDTBT should also present a layered structure similar to P3HT. In particular the presence of additives such as 1,8-diiodooctane (DIO) in the processing solvent induces these layered structures. ${ }^{17-21}$ Such structures were hypothesized from the presence of an in-plane reflection at $3.8 \AA$ and an out-of-plane reflection in the range $10.5 \AA-12.0 \AA$. Recent work showed that highly crystalline PCPDTBT films can be prepared by solvent vapor annealing (SVA) in chlorobenzene $(\mathrm{CB})$. The structure of these PCPDTBT crystals was analyzed by electron diffraction (ED) and a tentative model was proposed for the polymer without taking into account the side chains and using only the $h k 0$ single-crystal pattern. ${ }^{22}$ The extinction rules in this ED pattern were compatible with a $\mathrm{P}$ n $2 \mathrm{n}$ space group and four chains per unit cell. To our surprise, a packing mode similar to that of $\alpha$-PF8 and $\gamma$-PF7 could be evidenced with pairs of PCPDTBT chains packed in an orthorhombic unit cell. However, in the absence of ED patterns giving information along the chain direction, no precise packing scheme of the chains in the dimer could be proposed. In particular the type of stacking between cyclopentadithiophene and benzothiadiazole could not be clarified.

With respect to optical properties, it was found that the layered structures formed in the presence of DIO exhibit a strong absorption band around $800 \mathrm{~nm}$, which is attributed to $\pi$ stacking. ${ }^{17-21}$ The highly crystalline SVA films on the other hand exhibit a blue-shifted absorption and no absorption around $800 \mathrm{~nm} .^{22}$ 


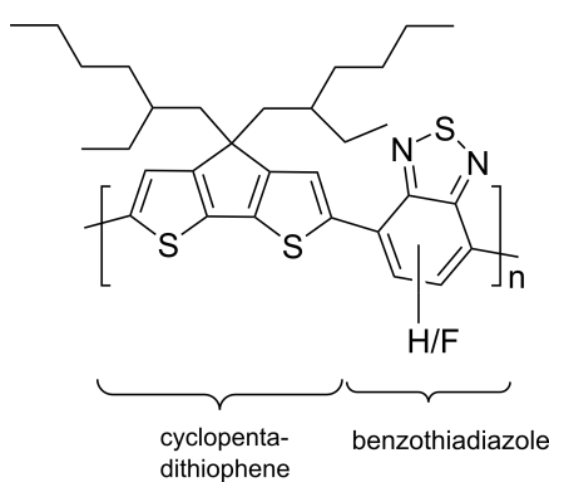

Scheme 1. Chemical structures of PCPDTBT and F-PCPDTBT.

This contribution presents new experimental evidence for polymorphism in the parent polymer PCPDTBT and its fluorinated derivative poly[2,6-(4,4-bis-(2-ethylhexyl)-4Hcyclopenta[2,1-b;3,4-b']dithiophene)-alt-4,7-(5-fluoro-2,1,3-benzothiadiazole)] F-PCPDTBT (in F-PCPDTBT, one hydrogen atom on the BT unit is replaced by fluorine regio-randomly, see scheme 1). From the point of view of device performance, the introduction of fluorine in the backbone of PCPDTBT results in improved efficiencies in OPV cells. ${ }^{23}$ To address the structure of these polymers, highly crystalline samples were prepared by SVA, leading to terrace-like domains with essentially standing chains and typical $\left[\begin{array}{lll}0 & 0 & 1\end{array}\right]$ ED patterns. Additional ED patterns were obtained from oriented films of these polymers prepared either by high-T rubbing or by epitaxy on oriented poly(tetrafluoroethylene) (PTFE) substrates. Absorption spectroscopy of the oriented films revealed a blue-shifted absorption without the $800 \mathrm{~nm}$ band, similar to the SVA samples. Two polymorphs are evidenced for both polymers and structural models of the form I are proposed. 


\section{Experimental section.}

a) Materials

Poly[2,6-(4,4-bis-(2-ethylhexyl)-4H-cyclopenta[2,1-b;3,4-b']dithiophene)-alt-4,7-(2,1,3benzothiadiazole)] (PCPDTBT) was purchased from 1-Material. The $\mathrm{M}_{\mathrm{w}}$ is $23 \mathrm{~kg} / \mathrm{mol}$ with a PDI of 1.7 as measured by HT-SEC $\left(160^{\circ} \mathrm{C}\right.$, trichlorobenzene, against PS standards). Poly[2,6-(4,4-bis-(2-ethylhexyl)-4H-cyclopenta[2,1-b;3,4-b']dithiophene)-alt-4,7-(5-fluoro2,1,3-benzothiadiazole)] (F-PCPDTBT) was synthesized by the group of S. Janietz at the Fraunhofer Institut für Angewandte Polymerforschung in Potsdam. A molecular mass of $\mathrm{M}_{\mathrm{w}}$ $=10.8 \mathrm{~kg} / \mathrm{mol}$ and a PDI of 1.6 was obtained by HT-SEC $\left(160^{\circ} \mathrm{C}\right.$, trichlorobenzene, against PS standards). All solvents (chlorobenzene, $\mathrm{CHCl}_{3}$,) were purchased from Sigma Aldrich (p.a. grade)

b) Solvent vapor annealing

Chlorobenzene (CB) solvent vapor annealing was employed to obtain highly crystalline samples of PCPDTBT and F-PCPDTBT. The detailed CB-annealing procedures for PCPDTBT have been described elsewhere. ${ }^{22}$ For the annealing procedure pre-cast films $\left(\mathrm{CHCl}_{3}, 3 \mathrm{mg} / \mathrm{ml}\right.$, spin coated) on glass substrates were used. The $\mathrm{CB}$ vapor atmosphere and the substrates were heated to $50^{\circ} \mathrm{C}$ and $40-42^{\circ} \mathrm{C}$, respectively and the films swollen to a solution-like state at a vapor pressure of $80-90 \%$. Recrystallization was subsequently induced by slowly decreasing the vapor pressure down to $73-70 \%$.

The CB-annealing method was slightly modified for F-PCPDTBT due to its stronger aggregation behavior which prevented a swelling of the pre-cast films. The substrates were covered inside the solvent vapor chamber with a CB solution $(0.1 \mathrm{mg} / \mathrm{ml})$ and equilibrated for 
10 min by purging the chamber with a $\mathrm{CB}$ vapor of $92 \%$ and holding the substrate and vapor at $42^{\circ} \mathrm{C}$ and $50^{\circ} \mathrm{C}$, respectively. During this time a thin homogenous film of the solution was formed which was afterwards recrystallized by reducing the vapor pressure to $74 \%$. Within 900 min the film was recrystallized completely and could be quenched by pure nitrogen.

c) Melt-crystallization on friction transferred PTFE substrates

Oriented PTFE substrates were prepared by friction transfer on clean glass slides using the protocol described in the literature. ${ }^{24}$ Thin polymer films $(3 \mathrm{mg} / \mathrm{ml}, \mathrm{CB})$ were cast on oriented PTFE substrates and melt-recrystallized in a Linkam hot stage (THMS 600) under argon atmosphere (melting at around $290^{\circ}$, cooling at $0.25 \mathrm{~K} / \mathrm{min}$ to $280^{\circ} \mathrm{C}$ for $4 \mathrm{hrs}$, final cooling at $0.25 \mathrm{~K} / \mathrm{min}$ to room temperature).

d) High temperature rubbing

High temperature rubbing was performed following the procedure described in the literature. ${ }^{12}$ For the rubbing experiments thin films were prepared by doctor-blading from a 8 $\mathrm{mg} / \mathrm{ml} \mathrm{CB}$ solution. For the alignment of the films, a home-made rubbing machine was used in a glove box (Plas-Labs Inc.) under nitrogen atmosphere. It is composed of a rotating cylinder ( $4 \mathrm{~cm}$ diameter) covered by a microfiber cloth. The rubbing is performed by applying the rotating cylinder with a 2 bar pressure on the translating sample holder $(1 \mathrm{~cm} / \mathrm{s})$. The sample holder can be heated at controlled temperatures during the rubbing process. The sample temperature is allowed to equilibrate for 1-2 min before rubbing. In the following, $\mathrm{R}$ designates the rubbing direction. Post-alignment thermal annealing was done in a THMS600 
Linkam hot stage under nitrogen atmosphere at the required temperature for 1 min. Heating and cooling rates were $20 \mathrm{~K} / \mathrm{min}$ and $0.5 \mathrm{~K} / \mathrm{min}$, respectively.

e) Structural analysis

TEM: Oriented areas were identified for TEM analysis by optical microscopy (Leica DMR-X microscope). The polymer films were coated with a thin amorphous carbon film and removed from the glass substrate by floating on a diluted aqueous HF solution (5 wt $\%$ ) and subsequently recovered on TEM copper grids. TEM was performed in bright field, high resolution and diffraction modes using a CM12 Philips microscope equipped with a MVIII (Soft Imaging System) Charge Coupled Device camera. Calibration of the reticular distances in the ED patterns was made with an oriented PTFE film.

Molecular modelling was performed on a Silicon Graphic station using the Cerius2 program (Accelrys). A trial-and-error procedure was used to refine the crystal structure of PCPDTBT and F-PCPDTBT, following the same methodology as used previously to refine the crystal structures of P3HT, PF8 and poly(3-hydroxy- $p$-methylphenylvalerate) $)^{15,25,26}$ as well as some conjugated co-oligomers ${ }^{27}$. For each step of the trial-and-error method, the molecular geometry was optimized using the "clean" procedure of the Cerius2 3.0 Program. The starting point of the CPDT-BT monomer structure was obtained from the published structure of the oligomer CPDT-BT-CPDT. ${ }^{28}$ For the single crystal diffraction calculations, a thickness of $30 \mathrm{~nm}$ was used. A $10 \%$ intensity threshold was used for the calculations of the fiber patterns using a precision symmetry (additional information on this procedure is available in the User's guide on Modeling Environment). 


\section{Results and discussion.}

\subsection{Lamellar crystals of form I of PCPDTBT and F-PCPDTBT.}

Solvent vapor annealing of PCPDTBT and F-PCPDTBT in CB, hereafter called CBannealed films, resulted in thin films with highly crystalline domains as illustrated in Figure 1. The polymorph obtained under these conditions is named form I for both polymers. Representative Selected Area Electron Diffraction (SAED) patterns of flat-on morphologies are shown in Figure 1.c and 1.d (the corresponding BF areas are shown in Figure 1.a and 1.b) for PCPDTBT and F-PCPDTBT respectively. PCPDTBT forms terrace-like flat-on features whereas F-PCPDTBT yields more elongated fiber-like flat crystals (Figure S2). (see BF image in Fig. 1.b). SAED yields for both polymers well defined ED patterns with high symmetry and rather sharp reflections. However, the F-PCPDTBT patterns always display sharper reflections than PCPDTBT. From the flat-on morphology of the diffracting areas, we infer that the ED patterns correspond to domains with standing chains, i.e. the ED patterns correspond to the $\left[\begin{array}{lll}0 & 0 & 1\end{array}\right]$ zone. Accordingly, both ED patterns can be indexed using the parameters : $a=12.4 \AA$ and $b=19.3 \AA$ for PCPDTBT and $a=11.0 \AA$ and $b=20.6 \AA$ for FPCPDTBT. 

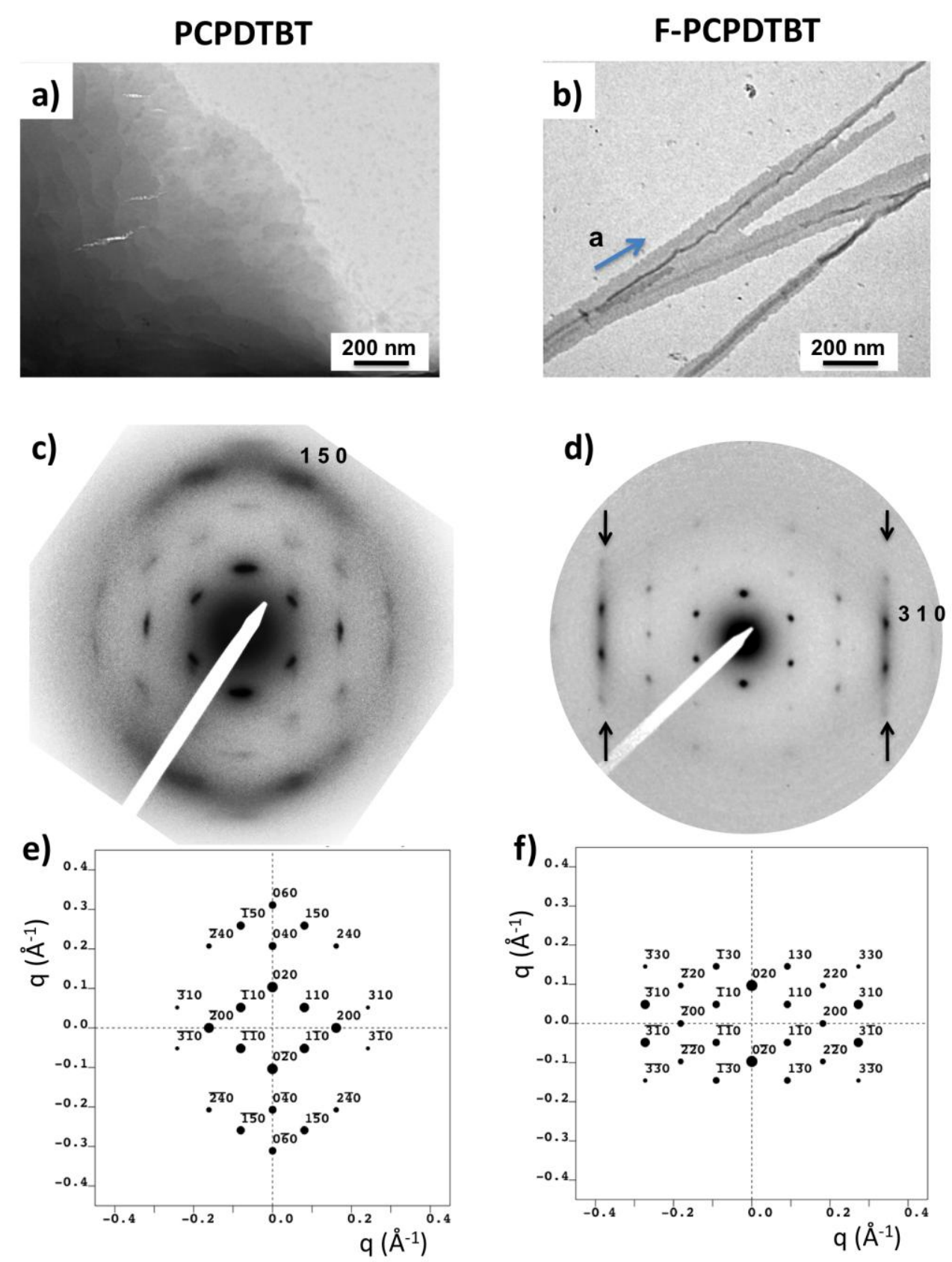

Figure 1. Bright field images (a, b), experimental ED patterns (c, d) and calculated ED patterns for the $\left[\begin{array}{lll}0 & 0 & 1\end{array}\right]$ zone (e, f) for PCPDTBT and F-PCPDTBT using the refined structural models shown in figure 4. The arrows in Figure 1.d highlight the streaked reflections in the ED pattern of the F-PCPDTBT crystals.

Overall the $\left[\begin{array}{lll}0 & 0 & 1\end{array}\right]$ patterns of F-PCPDTBT bear striking similarity with that of $\alpha$-PF8 crystals (see Figure 7 of reference 15). In $\alpha$-PF8, chains form dimers that are arranged in an 
orthorhombic unit cell. The corresponding pattern of PF8 reflects the following arrangement: it shows the reflections associated with the overall packing of the dimers in the unit cell

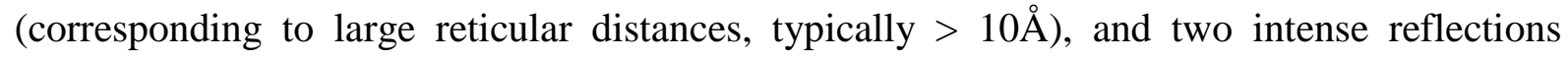
corresponding to the inter-chain distance in the dimer (around $5 \AA$ ). The ED patterns of PCPDTBT and F-PCPDTBT show similar characteristics. They consist of a set of 6 intense

reflections in a quasi-hexagonal lattice with reticular distances in the range of $9-11 \AA$ and a set of two intense reflections indexed as \pm 150 and \pm 310 for PCPDTBT and F-PCPDTBT, respectively. These characteristics suggest a high symmetry packing of chains into dimers with short 3.6-3.8 $\AA \pi$-stacking distance (in PF8 and PF7 no $\pi$-stacking is evidenced in the dimers). Although both polymers imply a pairing of chains into dimers, the arrangement of the dimers in the unit cell are different for F-PCPDTBT and PCPDTBT. First, both structures show slightly different unit cell parameters (see Table 1). Second, the intense reflections corresponding to the inter-chain distance in the dimer are differently indexed. As demonstrated in our structural modelling below, the plane of the conjugated backbone is parallel to the a-axis for PCPDTBT and to the $\mathbf{b}$-axis for F-PCPDTBT. This first result is remarkable and shows that the substitution of a single hydrogen atom by fluorine in the backbone of the polymer modifies significantly the packing of polymer chains in the crystal of form I. Further structural differences between PCPDTBT and F-PCPDTBT will be highlighted from the structural modeling (vide infra).

\subsection{In-plane orientation of PCPDTBT and F-PCPDTBT chains.}

To propose a valid structural model and in particular to establish the stacking mode between BT and CPDT in the dimers, characteristic fiber patterns showing multiple $00 \mathrm{l}$ reflections are needed. They were obtained from highly aligned films prepared by high-T rubbing or melt-crystallization of the polymers on friction transferred PTFE substrates. 
Figure 2.a and 2.c show BFs and the corresponding fiber-like patterns (Figure 2.b and d) of oriented PCPDTBT and F-PCPDTBT, respectively. Regular and periodic lamellar morphologies characteristic of edge-on oriented lamellae are evidenced for both polymers, regardless of the preparation method. Lamellar periods of $13 \mathrm{~nm}$ and $29 \mathrm{~nm}$ are obtained in FPCPDTBT films rubbed at $240^{\circ} \mathrm{C}$ and oriented PCPDTBT films on PTFE substrate, respectively.

The ED patterns in Figure 2 of both the rubbed F-PCPDTBT and the epitaxied PCPDTBT film on PTFE are very detailed with numerous layer lines (up to the $10^{\text {th }}$ ). For each of the two polymers, the observed reflections can be clearly attributed to the form I evidenced in the CB-annealed films. In F-PCPDTBT, three major reflections of form I appear on the meridian: the 020 reflection at $10.55 \AA$, a 110 reflection at $9.7 \AA$ and the 310 reflection at $3.7 \AA ̊$ (Supporting Figure S1). In PCPDTBT, two dominant equatorial reflections of form I are observed: the 110 at $10.5 \AA$ and the 150 at $3.7 \AA$. The presence of several $h k 0$ reflections on the equator indicates that the rubbed and epitaxied films have a typical fiber symmetry such that $\mathrm{c}_{\mathrm{PCPDTBT}} / / \mathrm{c}_{\mathrm{PTFE}}$ and $\mathrm{c}_{\mathrm{F}-\mathrm{PCPDTBT}} / / \mathrm{R}$. 
Table 1. Main reflections of the fiber patterns of PCPDTBT and F-PCPDTBT and corresponding indexation using the orthorhombic unit cell of form I .

\begin{tabular}{|c|c|c|}
\hline Indexation (Form I) & PCPDTBT & F-PCPDTBT \\
\hline$\left(\begin{array}{lll}1 & 1 & 0\end{array}\right)$ & $10.5 \AA$ & $9.7 \AA$ \\
$\left(\begin{array}{lll}0 & 2 & 0\end{array}\right)$ & - & $10.55 \AA$ \\
$\left(\begin{array}{lll}0 & 1 & 2\end{array}\right)$ & $10.0 \AA$ & $10.03 \AA$ \\
$\left(\begin{array}{lll}0 & 0 & 4\end{array}\right)$ & $5.9 \AA$ & $5.8 \AA$ \\
$\left(\begin{array}{lll}1 & 0 & 6\end{array}\right)$ & $3.75 \AA$ & - \\
$\left(\begin{array}{lll}1 & 5 & 0\end{array}\right)$ & $3.7 \AA$ & $3.69 \AA$ \\
$\left(\begin{array}{lll}3 & 1 & 0\end{array}\right)$ & - & \\
\hline
\end{tabular}



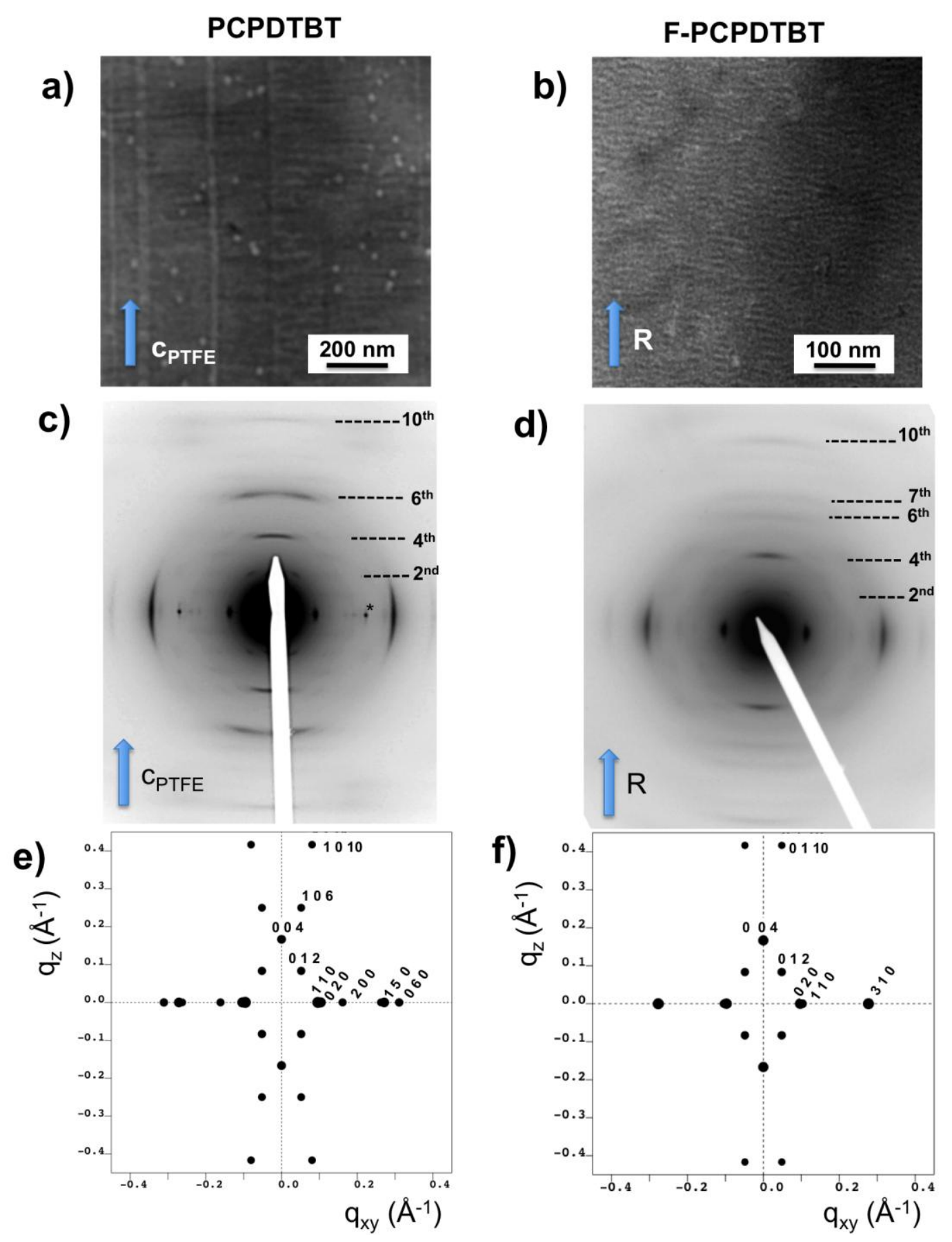

Figure 2. Bright field images (a and b), experimental ED patterns (c and d) and calculated ED patterns (e and f) for highly oriented films of PCPDTBT and F-PCPDTBT with a fiber-like symmetry. The films of PCPDTBT were prepared by melt-crystallization on frictiontransferred PTFE (the PTFE chain axis direction is indicated by an arrow) whereas the films of F-PCPDTBT are prepared by high-T rubbing at $240^{\circ} \mathrm{C}$ (the rubbing direction $\mathrm{R}$ is indicated by an arrow). In part (c), the asterisk highlights the 100 reflection of PTFE. 
Of importance for the subsequent structural analysis is the intensity distribution of the reflections along the chain direction. Along the meridian of the ED patterns, strong reflections are observed on the $4^{\text {th }}, 6^{\text {th }}$ and $10^{\text {th }}$ layer lines of PCPDTBT and on the $4^{\text {th }}$ and $10^{\text {th }}$ layer line of F-PCPDTBT (a few more diffuse and weak reflections exist on intermediate layer lines). In both patterns, the most intense meridional reflection is observed at $5.9 \AA$ in PCPDTBT and 5.8 $\AA$ in F-PCPDTBT. It corresponds to the 004 reflection of form I. Thus, the patterns allow to extract the $\mathbf{c}$ axis parameter for both polymers: $c=23.6 \pm 0.5 \AA$ for PCPDTBT and $c=23.2 \pm 0.5 \AA$ for F-PCPDTBT. Most importantly, no $\left(\begin{array}{lll}0 & 0 & 2 n+1)\end{array}\right)$ reflection is present on the meridian.

To conclude, the oriented fiber patterns of both polymers yield additional important structural informations that will be used for the subsequent structural refinement of form I dealing with the relative arrangement of the polymer chains along the chain direction. Moreover, they allow to complete the unit cell parameters of form I determined from the $[00$ 1] zones in CB-annealed samples. Table 2 summarizes the unit cell parameters for the form I of F-PCPDTBT and PCPDTBT.

Table 2. Lattice parameters for the orthorhomic form I of PCPDTBT and F-PCPDTBT and their calculated densities $r_{\text {calc }} \mathrm{Z}$ is the number of repetition units (CPDTBT) per unit cell.

\begin{tabular}{|c|c|c|c|c|c|}
\hline Polymer/ & $\mathrm{a}(\AA)$ & $\mathrm{b}(\AA)$ & $\mathrm{c}(\AA)$ & $\mathrm{Z}$ & $\rho_{\text {calc }}\left(\mathrm{g} / \mathrm{cm}^{3}\right)$ \\
parameters & $( \pm 0.3 \AA)$ & $( \pm 0.3 \AA)$ & $( \pm 0.3 \AA)$ & & \\
\hline PCPDTBT & 12.4 & 19.3 & 23.6 & 8 & $1.08 \pm 0.05$ \\
F-PCPDTBT & 11.0 & 20.6 & 23.2 & 8 & $1.18 \pm 0.05$ \\
\hline
\end{tabular}




\subsection{Absorption spectra of different polymorphs.}

The previous structural investigations by ED indicate that form I of PCPDTBT and FPCPDTBT can be generated either by solvent vapor annealing in $\mathrm{CB}$, high-T rubbing and melt-crystallization on PTFE substrates, although with different morphologies and orientations of crystalline domains on the substrates. UV-vis absorption spectroscopy is used to further confirm this result.

The absorption spectrum of form I of PCPDTBT is characterized by two dominant components at $680 \mathrm{~nm}$ and $740 \mathrm{~nm}$ (see Figure 3a, blue line). ${ }^{22}$ The melt-annealed PCPDTBT film on PTFE shows a very similar absorption spectrum (see Figure 3 a, black line). On the other hand, films prepared by following the device processing protocol from literature, i.e. spin coating from $\mathrm{CB}$ solution with $2 \mathrm{w} \%$ DIO as solvent additive (hereafter called CB/DIOfilms), reveal a strong $800 \mathrm{~nm}$ band and a shoulder at $740 \mathrm{~nm}$. In the literature ${ }^{18-20}$, the $800 \mathrm{~nm}$ band has been correlated with the presence of long range $\pi$-stacking within an edgeon morphology, in analogy to P3HT. Temperature-dependent absorption and photoluminescence spectra and a detailed Franck-Condon analysis proved the aggregated nature of these films. ${ }^{29}$ We suggest that the absence of the $800 \mathrm{~nm}$ absorption band in form I films can be explained by the absence of any long-range $\pi$-stacking in the CB-annealed and the melt-annealed aligned films. The structural analysis further supports this finding (vide infra).

In the case of F-PCPDTBT, the same trend is visible: CB/DIO-films show a distinct absorption maximum at $800 \mathrm{~nm}$ whereas both the spectrum of the CB-annealed and the high temperature rubbed films are notably blue-shifted (see Figure 3). This suggests that two polymorphs can also be obtained in F-PCPDTBT: one that is described in the literature as due to $\pi$-stacking and form I which is comparable to PCPDTBT. In literature all absorption 
spectra of F-PCPDTBT films show the characteristic $800 \mathrm{~nm}$ absorption. It is suggested that in F-PCPDTBT the $800 \mathrm{~nm}$ band is also correlated to an edge-on morphology comprising a long range $\pi$-stacking similar to that in PCPDTBT. ${ }^{23}$ This is further supported by GIWAXSresults by Jen et al. ${ }^{30}$ Interestingly, simple spin coating from CB already results in similar spectra as the $\mathrm{CB}$ / DIO-films. It was suggested that this is due to a stronger stacking tendency of F-PCPDTBT as compared to PCPDTBT. ${ }^{23}$

Both TEM and UV-vis spectroscopy demonstrate that several preparation protocols (melt-annealing, HT-rubbing and solvent vapor annealing) all lead to the same polymorph, i.e. form I which involves pairing of chains into dimers. The fact that slow crystallization methods such as melt-crystallization or solvent vapor annealing lead to the form I polymorph suggests that it is the thermodynamically stable structure and not a solvent-induced structure.

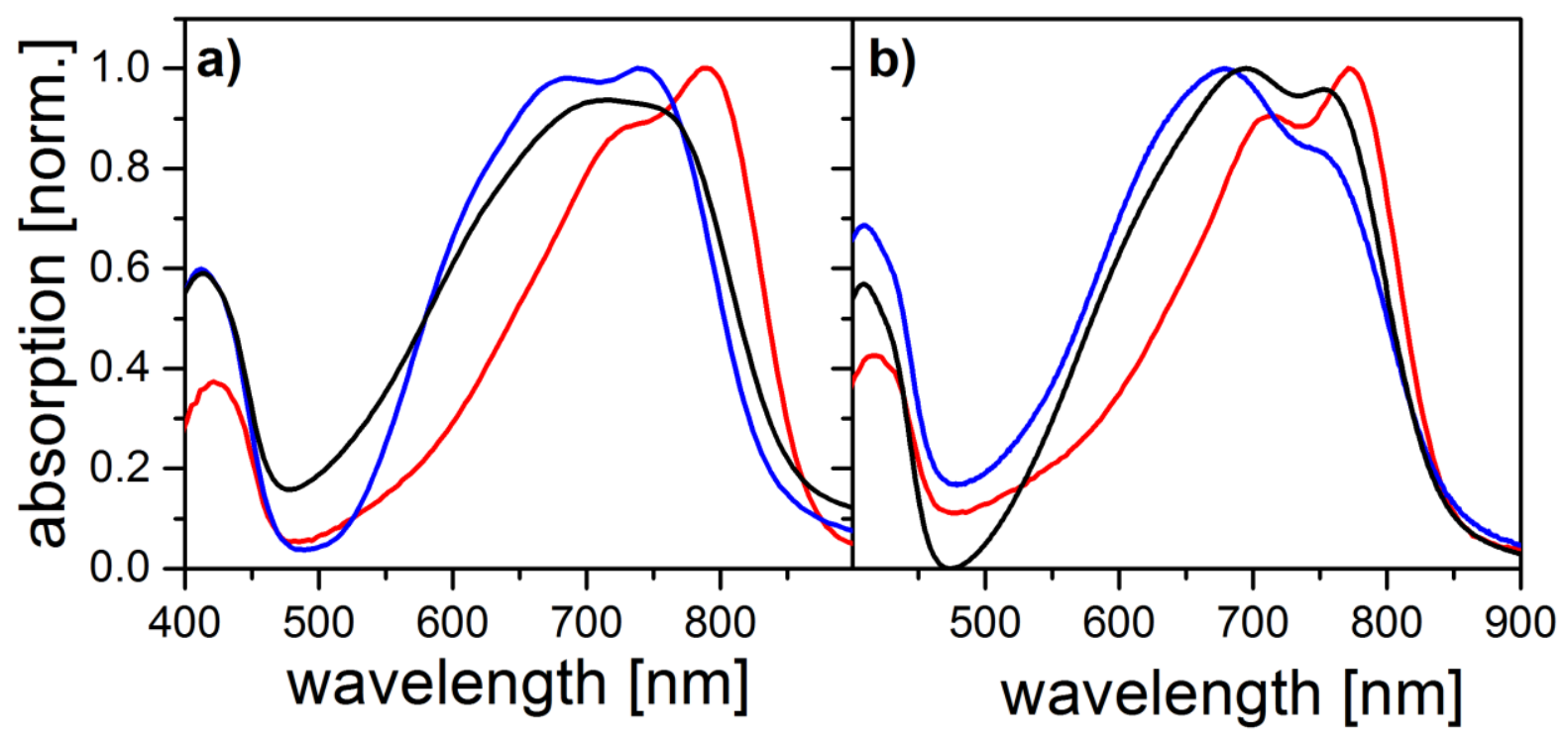

Figure 3 Absorption spectra of thin films prepared by different techniques. a) PCPDTBT absorption spectra of thin films prepared by spin coating from $\mathrm{CB} / \mathrm{DIO}$ (2w\%, red), CB-annealing (blue) and melt-annealing on PTFE (black). b) F-PCPDTBT absorption spectra of thin films prepared by spin coating from $\mathrm{CB} / \mathrm{DIO}\left(2 \mathrm{w} \%\right.$, red), $\mathrm{CB}$-annealing (blue) and high temperature rubbing $\left(240^{\circ} \mathrm{C}\right.$, black). 


\subsection{Structural models of form $I$.}

First, it is worth to stress that, although sophisticated methods of crystallization were used for both polymers, the number of reflections extracted from the ED patterns is much more limited than for $\pi$-conjugated polymers such as P3HT or polyfluorenes (PFO and PF2/6). This sets a strong limitation on the details that can be extracted from the ED patterns. For instance, the exact side chain conformation cannot be determined precisely but only an approached conformation that yields the required electronic density distribution in the unit cell to properly account for the observed ED patterns. The side chain conformation shown in the refined models is only tentative but must be included in order to reproduce correctly the intensities in the $00 l$ layers of the fiber patterns. In addition, the structural models use only one conformation of the 2-ethylhexyl side chain whereas four stereoisomers of the CPDT monomer (SS, RR, and two SR) should be present in the chains. For the structural modeling, we have chosen arbitrarily the SS conformation of the monomer (the choice of the stereoisomer was found to impact only marginally the calculated intensities of the reflections in the ED patterns).

In our previous work ${ }^{22}$, a first tentative model of form I PCPDTBT without sidechains was proposed based on the sole [llll $\left.0 \begin{array}{ll}0 & 1\end{array}\right]$ pattern, i.e. with no information on the relative arrangement of the chains along the chain direction contained in the fiber patterns. With the addition of the newly obtained fiber patterns, the different ED patterns can be used for the determination of the space group by establishing the selection (extinction) rules. For the form I of both polymers, the same set of selection rules was determined: 
(h 00 ) with $\mathrm{h}=2 \mathrm{n}$

(0 k 0) with $\mathrm{k}=2 \mathrm{n}$,

(h k 0) with $\mathrm{h}+\mathrm{k}=2 \mathrm{n}$ and

$\left(\begin{array}{lll}0 & 0 & 1\end{array}\right)$ with $1=2 n$.

These rules are compatible with several orthorhombic space groups. To mention but a few: P n 2 n, P n c n, C c c 2 and P c c n. ${ }^{31}$ The first space group generates 4 asymmetric units in a unit cell contrary to 8 for the latter two. Given the refined unit cell parameters, 8 asymmetric units, i.e. 8 monomers must be present in the unit cell to obtain a reasonable crystal density around $1 \mathrm{~g} / \mathrm{cm}^{3}$. Therefore, the space groups with superior symmetry P $\mathrm{n} \mathrm{c} \mathrm{n,} \mathrm{C}$ c c 2 and P c c n are considered hereafter (the lower symmetry group P n 2 n group was retained in our previous study because, in the absence of information along the chain direction, a set of two monomers in trans conformation was used). The fiber patterns allow to further discriminate between these three space groups. Indeed, in the case of the C c 2 group, the arrangement of the chains in the unit cell corresponds to a segregated stacking of BZ and CPDT units (see Figure S3). In C c c 2 symmetry, the relative arrangement of the chains in the unit cell is such that the alkyl chains are located around 002 planes. Such a packing would result in a strong 002 reflection in the fiber patterns. However, the experimental fiber patterns do not show any 002 reflection, thus ruling out the $\mathrm{C}$ c c 2 space group. Therefore, only the P n c n and P c c n space groups are retained. These two space groups lead to two radically different molecular packings of the chains in the dimers as illustrated in Figure S3. Several arguments allow to retain the dimer conformation obtained for the P c c n space group, i .e. the segregated stacking mode of BT and CPDT. First, the models with a $\mathrm{P} n \mathrm{c} n$ symmetry leading to the best agreeement between calculated and experimental ED patterns correspond to a mixed stacking of BT and CPDT units which 
results in unrealistic short interatomic contacts between the chains in the dimer. Second, a strong argument for the proposed model with P c c n symmetry concerns the arrangement of BTs in the dimers. A recent study on a CPDT-BT-CPDT oligomer has demonstrated a faceto-face stacking of the BT units. ${ }^{28}$ The choice of the $\mathrm{P} \mathrm{c} \mathrm{c} \mathrm{n}$ space group is further important since it allows the backbones of PCPDTBT and F-PCPDTBT to $\pi$-stack despite the presence of the sterically-demanding 2-ethylhexyl side chains.

Figure 4 shows the projections of the refined crystal structures of PCPDTBT and FPCPDTBT along the three axes of the unit cell that correspond to the best agreement between experimental and calculated ED patterns ( $h k 0$ and fiber). The comparison between experimental and calculated patterns indicates a very good agreement despite small discrepancies such as the overestimation of the 040 intensity for PCPDTBT. Clearly, FPCPDTBT shows the best agreement for the $h k 0$ pattern. The agreement observed for the fiber patterns is remarkable: the absence of strong reflections in the $6^{\text {th }}$ layer line in FPCPDTBT is indeed well reproduced. As a test for the sensitivity of our modeling to the presence/absence of the side chains, we compare in Figure S4 the calculated $\left[\begin{array}{lll}0 & 0 & 1\end{array}\right]$ patterns for F-PCPDTBT for the model with and without the alkyl side chains. Clearly, the dominant features in the $\left[\begin{array}{lll}0 & 0 & 1\end{array}\right]$ pattern are observed in both models of F-PCPDTBT i.e. with and without including the side chains. However, the correct simulation of the fiber patterns requires to include the side chains since they contribute importantly to the scattered intensity in the $1^{\text {st }}$ layer lines.

Let us analyze the major structural features of both models. First, we observe that the backbones of both PCPDTBT and F-PCPDTBT can be considered as planar although a small dihedral angle between the planes of CPDT and BT might be possible (in the CPDT-BTCPDT oligomer, the BT and CPDT units are not perfectly copolanar ${ }^{28}$ ). Second, for both polymers, the chains form dimers with a segregated stacking of CPDT and BT. The evidence 
of a specific pairing of PCPDTBT and F-PCPDTBT chains into dimers with segregated stacking is an interesting finding from several points of views. First, such a dimerization prevents long-range $\pi$-stacking as observed in semi-conducting polymers such as P3HT or PBTTT for which chains can $\pi$-stack over large length scales (for instance in micrometer-long crystalline nanofibrils formed in $p$-xylene or anisole). This should also have implications on charge transport in such polymers. Second, the dimers are packed in an orthorhombic unit cell with $b \approx 2 a$ i.e. close to a hexagonal packing. This suggests that dimers may form first in solution and assemble afterwards in a hexagonal lattice when the films are in a swollen state in the presence of chlorobenzene. Finally, upon deswelling of the films, the unit cell becomes orthorhombic because of the side chain ordering. It is in this final step that the structures of FPCPDTBT and PCPDTBT differ. Despite fairly close unit cell parameters, a remarkable difference in the packing of the dimers in the unit cells of PCPDTBT and F-PCPDTBT is observed: the backbone plane is parallel to $(\mathbf{a}, \mathbf{c})$ in PCPDTBT and $(\mathbf{b}, \mathbf{c})$ in F-PCPDTBT. It is however yet unclear how such a strong difference in the packing of the dimers can be linked to the presence/absence of a single fluorine atom in the backbone of the polymer.

The $\left[\begin{array}{lll}0 & 0 & 1\end{array}\right]$ experimental pattern of F-PCPDTBT shows a characteristic streaking along $3 \mathrm{k} 0$ (see Figure 1.e) (mainly visible for the 310 reflection). Similar streaked features were observed previously in single crystal ED patterns of syndiotactic polypropylene (sPP) under certain crystallization conditions and/or tacticity of the polymer. Streaking in sPP crystals is observed along $h 10$. It is characteristic of stacking defects corresponding either to extended rows of chains displaced by $b / 4$ or to groups of such rows displaced by $b / 4 .{ }^{32,33}$ In FPCPDTBT, the streaking is observed essentially for the 310 and 330 reflections. It is absent from other layer lines. Therefore, it is proposed that this streaking arises from disorder in the chain arrangement within the dimers rather than disorder in the packing of the dimers in the crystal. In PCPDTBT structural disorder does not result in streaking but rather in a blurring of 
the 150 and 420 reflections. It is considered as a fingerprint of structural disorder within the PCPDTBT dimers.

Disorder in the arrangement of PCPDTBT and F-PCPDTBT is not unexpected when considering the recent structural investigations on a cyclopentadithiophene-benzothiadiazole oligomer. ${ }^{28}$ Indeed, the thiadiazole tip of the BT unit was found to point equiprobably in two possible directions in the crystal of this oligomer. In the case of F-PCPDTBT, this would mean that the sulfur tip of BT could point either along $\mathbf{b}$ or $-\mathbf{b}$ in the absence of strong steric hindrance by the surrounding alkyl side chains. A further source of disorder in both polymers is related to the coexistence of four stereoisomers (RR, RS, SR and SS) of CPDT since the 2ethylhexyl side chain contains an asymmetric carbon. These four stereoisomers are expected to occur at random in the chains, leading expectedly to some packing disorder of the chains within the dimers. 

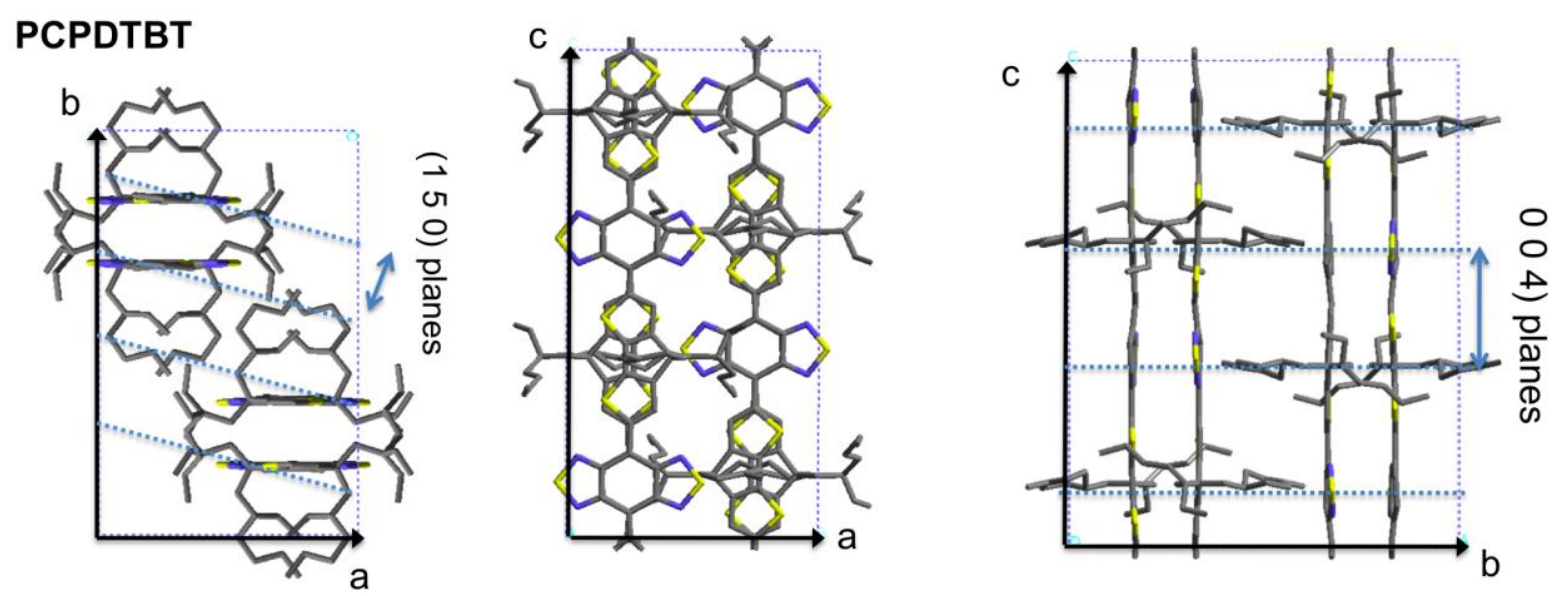

\section{F-PCPDTBT}
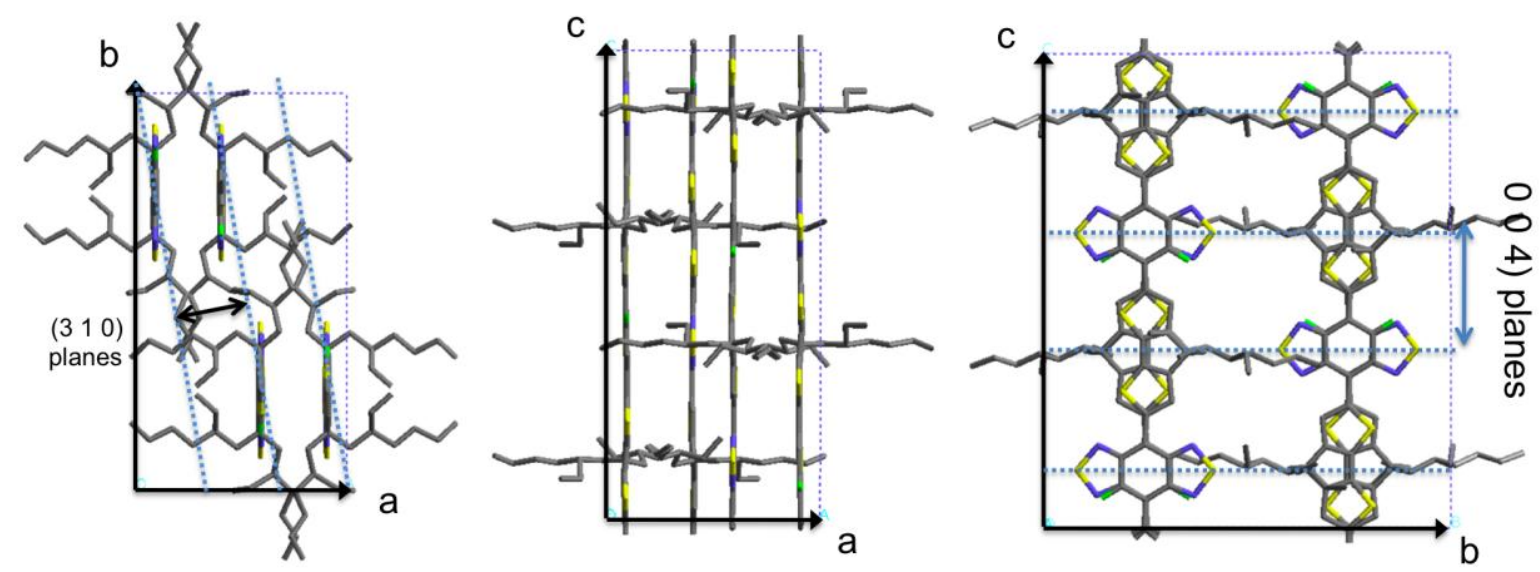

Figure 4. Projections along the a,b and c unit cell axes of the structural models refined from the ED data shown in figure 1 and 2 for PCPDTBT and F-PCPDTBT.

\subsection{Evidence for a further polymorph on PTFE substrates.}

Close examination of the films of PCPDTBT and F-PCPDTBT prepared by casting from $\mathrm{CB}$ on PTFE substrate, and annealing at $300^{\circ} \mathrm{C}$ for $4 \mathrm{hrs}$ and slow cooling to room temperature (with $0.25 \mathrm{~K} / \mathrm{min}$ ), show almost non-absorbing mm-large crystalline features (see Figure 5) beside the uniform areas corresponding to oriented form I (showing a strongly polarized light absorption as seen in the POM of figure 5).

Electron diffraction of these zones reveals a characteristic hexagonal-like pattern with two strong reflections as $7.55 \AA$ and $7.25 \AA$ for both PCPDTBT and F-PCPDTBT. Since these 
reflections cannot be indexed with the cell parameters of form I, they are attributed to the [0 0 1] zone of a different orthorhombic polymorph (named form II hereafter). The unit cell parameters for both polymers are almost identical with $a=8.3 \AA$ and $b=15.1 \AA$. From these two cell parameters, it can be stated that only two chains are contained in the unit cell, contrary to form I. Moreover, the intensities of the reflections do not give any evidence for the formation of dimers. It is therefore a novel polymorph clearly distinct from form I and literature.

Attempts to realize rotation-tilt on form II areas to obtain additional projections were not successful given the large thickness of the domains. Further structural investigations will be necessary to clarify the growth conditions leading exclusively to this polymorph. Oriented fiber patterns of form II would help further refine the crystal packing and clarify the type of stacking between BT and CPDT.

A) PCPDTBT

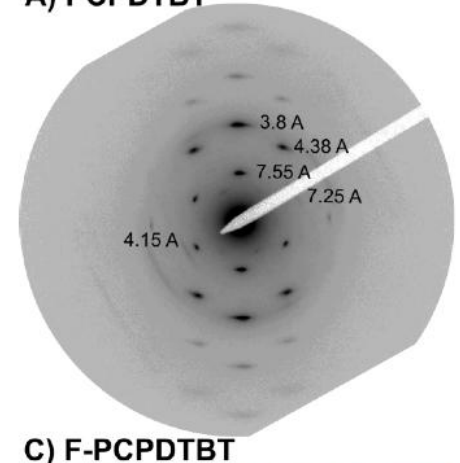

C) F-PCPDTBT

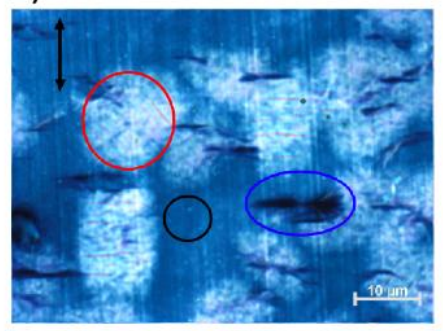

B) F-PCPDTBT
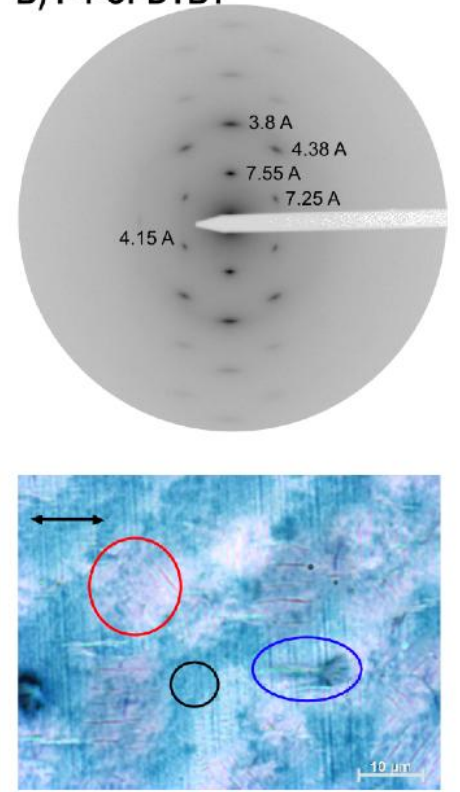

Figure 5. a) and b) show ED patterns taken from the highly crystalline almost transparent areas shown in the transmission polarized microscopy images of F-PCPDTBT in Figure $\mathrm{c}$ and 
d. The arrows in the top left corner of the images indicate the polarizer orientation. The red circle highlights the almost non-absorbing regions compared to the regions containing form I (blue circle) and the rather amorphous regions (black circle).

\section{Conclusion.}

Two distinct polymorphs (form I and form II) for PCPDTBT and F-PCPDTBT have been observed that differ from the structure observed in spin coated films from CB solution with $2 \mathrm{w} \%$ DIO that are used in photovoltaic devices. The refined structures of form I are distinct from the classical layered structure where layers of $\pi$-stacked backbones alternate with layers of alkyl side chains. In addition to form I, electron diffraction gives evidence for a second polymorph (form II) which is a priori isostructural for PCPDTBT and F-PCPDTBT. Form I has been obtained by various crystallization methods, i.e. SVA, melt-crystallization on oriented PTFE or high-T rubbing, indicating that it is a thermodynamically stable structure that is not induced by a specific solvent. The characteristic UV-vis absorption signature of this stable form has been determined for both polymers. The $h k 0$ and fiber-like ED patterns were used to generate tentative structural models of form I for both polymers. Form I involves pairing of chains into dimers arranged in an orthorhombic lattice (space group P c c n) with a pseudo-hexagonal symmetry. For both, PCPDTBT and F-PCPDTBT, a segregated stacking of BT and CPDT units is uncovered with BT units in anti-parallel face-to-face configuration. Our modeling suggests that the backbones of PCPDTBT and F-PCPDTBT are essentially planar, although a small twist between successive monomers cannot be fully excluded. Despite fairly similar unit cell parameters, the dimers of PCPDTBT and F-PCPDTBT are differently oriented in the unit cells leading to two distinct structures. These results demonstrate that a single $\mathrm{H} / \mathrm{F}$ substitution in the backbone of a conjugated polymer can 
significantly affect the chain packing in the crystal structure. As opposed to form I, the unit cell of form II contains two chains that are not paired as dimers.

\section{ASSOCIATED CONTENT}

\section{Supporting information.}

Enlarged electron diffraction pattern of F-PCPDTBT, microscopy images of CB-annealed FPCPDTBT samples, comparison of the stacking of CPDT and BTZ unit in a dimer of PCPDTBT for the structures with different space groups, comparison of the calculated [0 001$]$ patterns for the form I of F-PCPDTBT taking or not taking into account the 2-ethylhexyl side chains. PDB files of the refined structural models of PCPDTBT and F-PCPDTBT.

\section{AUTHOR INFORMATION}

Corresponding authors:

* E-mail : $\underline{\text { Martin.brinkmann@ics-cnrs.unistra.fr }}$

sabine.ludwigs@ipoc.uni-stuttgart.de

${ }^{+}$Present Address: College of Nanotechnology, King Mongkut's Institute of Technology Ladkrabang (KMITL), Bangkok, 10520, Thailand

\section{ACKNOWLEDGMENTS.}

M. B. acknowledges financial support by the European commission under contract Interreg Rhin-Solar (C25). We thank Silvia Janietz for synthesizing and giving us the F-PCPDTBT material. P. Martinoti is acknowledged for supplying the Plas-Labs glovebox. M. Schmutz and C. Blanck are acknowledged for technical support in TEM. Bernard Lotz is gratefully acknowledged for fruitful discussions. FF, DT and SL thank the DFG for funding within the priority program "Elementary processes in organic solar cells" (SPP1355) and the Emmy Noether Program. 


\section{References.}

(1) Zhang, Z.-G. and Wang, J. J. Mater. Chem. 2012, 22, 4187.

(2) Boudreault, P.-L. T. ; Najari, A. and Leclerc, M. Chem. Mater. 2011, 23, 456.

(3) Facchetti, A. Materials Today, 2013, 16, 123.

(4) Kularatne, R. S. ; Magurudeniya, H. D. ; Sista, P. ; Biewer, M. C. and Stefan, M. C. J. Polym. Sci. Polym. Chem. A 2013, 51, 743.

(5) Brinkmann, M. ; Gonthier, E. ; Bogen, S. ; Tremmel, K. ; Ludwigs, S. ; Hufnagel, M. ; Sommer, M. ACS Nano, 2012, 6, 10319.

(6) Donley, C. L. ; Zaumseil, J. ; Andreasen, J. W. ; Nielsen, M. M. ; Sirringhaus, H. ; Friend, R. H. ; Kim, J.-S. J. Am. Chem. Soc. 2005, 127, 12890-12899.

(7) W. Porzio, W. ; Scavia, G. ; Barba, L. ; Arrighetti, G. and McNeill, C.R. Euro. Pol. J. 2014, 61, 172-185.

(8) Crossland, E. J. W. ; Tremel, K. ; Fischer, F. S. U. ; Rahimi, K. ; Reiter, G. ; Steiner, U. and Ludwigs, S. Adv. Mater. 2012, 24, 839

(9) Crossland, E. J. W. ; Rahimi, K. ; Reiter, G. ; Steiner, U. and Ludwigs, S. Adv. Funct. Mater. 2011, 21, 518.

(10) (a) DeLongchamp, D. M.; Kline, R. J.; Jung, Y.; Germack, D. S.; Line, E. K. ; Moad, A. JL. J. Richter, L. J. ; Toney, M. F.; Heeney, M. and McCulloch, I. ACS Nano, 2009, 3, 780-787. (b) Lee, M. J.; Gupta, D.; Zhao, N.; Heeney, M.; McCulloch, I. and Sirringhaus, H. Adv. Funct. Mat. 2011, 21, 932. 
(11) (a) Müller, C. ; Aghamohammadi, M. ; Himmelberger, S. ; Sonar, P. ; Garriga, M. ; Salleo, A. and Campoy-Quiles, M. Adv. Funct. Mater. 2013, 23, 2368. (b) Brinkmann, M. and Wittmann, J.-C. Adv. Mat. 2006, 18, 860. (c) Brinkmann, M. ;Contal, C. ; Kayunkid, N. ; Djuriç, T. and Resel, R. Macromolecules 2010, 43, 7604. (d) Brinkmann, M. ; Charoenthai, N.; Traiphol, R. ; Piyakulawat, P. ; Wlosnewski, J. and Asawapirom, U. ; Macromolecules, 2009, 42, 8298.

(12) (a) Hartmann, L.; Tremel, K.; Uttiya, S.; Crossland, E.; Ludwigs, S.; Kayunkid, N.; Vergnat, C.; Brinkmann, M. Adv. Funct. Mat. 2011, 21, 4047-4057. (b) Biniek, L.; Leclerc, N.; Heiser, T. and Brinkmann, M. Macromolecules 2013, 46, 4014. (c) Biniek, L.; Poujet, S.; Djurado, D.; Gonthier, E.; Tremel, K.; Kayunkid, N.; Zaborova, E.; Crespo-Monteiro, N.; Boyron, O.; Leclerc, N.; Ludwigs, S. and Brinkmann, M. Macromolecules, 2014, 47, 3871. (d) Tremel, K. ; Fischer, F. S. U. ; Kayunkid, N. ; DiPietro, R. ; Kiriy, A. ; Neher, D. ; Ludwigs, S. and Brinkmann, M. Advanced Energy Materials, 2014, 4, 1301659. (e) Brinkmann, M. ; Hartmann, L. ; Biniek, L. ; Tremel, K. and Kayunkid, N. Macromol. Rapid. Comm. 2014, 35, 9.

(a) Tremel, K. and Ludwigs, S. Advances in Polymer Science 2014, 265, 39. (b) Brinkmann, M. J. Polym. Sci. Polym. Phys. 2011, 49, 1218.

(14) McCulloch, I.; Heeney, M.; Bailey, C.; Genevicus, K.; MacDonald, I.; Shkunov, M.; Sparrowe, D.; Tierney, S.; Wagner, R.; Zhang, W.; Chabinyc, M. L.; Kline, J.; McGehee, M. D.; Toney, M. F. Nat. Mater. 2006, 5, 328-333. Brinkmann, M. Macromolecules, 2007, 40, 7532.

(16) Knaapila, M.; Torkkeli, M. ; Galbrecht, F. and Scherf, U. Macromolecules 2013, 46, 834. 
(17) Rogers, J. T. ; Schmidt, K.; Toney, M. F.; Kramer, E. J. and Bazan, G. C. Adv. Mater. 2011, 23, 2284.

(18) Agostinelli, T.; Ferenczi, T. A. M.; Pires, E.; Foster, S.; Maurano, A.; Müller, C.; Ballantyne, A.; Hampton, M.; Lilliu, S.; Campoy-Quiles, M.; Azimi, H.; Morana, M.; Bradley, D. D. C.; Durrant, J.; Macdonald, J. E.; Stingelin, N.; Nelson, J.; J. Polym. Sci. Part B Polym. Phys. 2011, 49, 717.

(19) Gu, Y.; Wang, C. and Russell, T. P. Adv. Energy Mater. 2012, 2, 683.

(20) Peet, J. ; Cho, N. S. ; Lee, S. K. and Bazan, G. C. Macromolecules 2008, 41, 8655.

(21) Peet, J. ; Kim, J. Y. ; Coates, N. E. ; Ma, W. L. ; Moses, D. ; Heeger, A. J. and Bazan, G. C. Nat. Mater. 2007, 6, 497.

(22) Fischer, F. S. U. ; Trefz, D. ; Back, J. ; Kayunkid, N. ; Tornow, B. ; Albrecht, S. ; Yager, K. G. ; Singh, G. ; Karim, A. ; Neher, D. ; Brinkmann, M. and Ludwigs, S. Adv. Mater. 2015, 27, 1223.

(23) (a) Zhang, Y.; Zou, J.; Cheuh C.-C.; Yip H.-L. and Jen, A. J.-Y. Macomolecules 2012, 45, 5427, (b) Albrecht, S.; Janietz, S.; Schindler, W.; Frisch, J.; Kurpiers, J.; Kniepert, J.; Inal, S.; Pingel, P.; Fostiropoulos, K.; Koch, N. and Neher, D. J. Am. Chem. Soc. 2012, 134, 14932.

(24) Brinkmann, M. ; Graff, S. ; Wittmann, J.-C. ; Chaumont, C. ; Nuesch, F. ; Anver, A. ; Schaer, M. and Zuppiroli, L. J. Phys. Chem. B 2003, 107, 10531.

(25) Kayunkid, N. ; Uttiya, S. and Brinkmann, M. Macromolecules 2010, 43, 4961

(26) Hany, R. ; Brinkmann, M. ; Ferri, D. ; Hartmann, R. ; Pletscher, E. ; Rentsch, D. and Zinn, M. Macromolecules, 2009, 42, 6322.

(27) Biniek, L.; Schwartz, P.-O.; Zabrova, E.; Heinrich, B.; Leclerc, N.; Méry, S. and Brinkmann, M. J. Mater. Chem. C, 2015, 3, 3342. 
(28) Chang, S. W.; Waters, H.; Kettle, J. and Horie, M. Org. Electr. 2012, 13, 2967.

(29) Scharsich, C.; Fischer, F. S. U.; Wilma, K.; Hildner, R.; Ludwigs, S. and Köhler, A. Manuscript submitted

(30) Li, Y.; Zou, J.; Yip, H.-L.; Li, C.-Z., Zhang, Y.; C.-C. Chueh, Intemann, J.; Xu, Y.; Liang, P.-W., Chen, Y. and Jen, Alex K.-Y. Macromolecules 2013, 46, 5497.

(31) International Tables for Crystallography, Vol. A, Kluwer Academic Pub. 1989, p. 82 274.

(32) Lotz, B.; Lovinger, A. J. and Cais, R. E. Macromolecules 1988, 21, 2375.

(33) Lovinger, A. J.; Lotz, B. , Davis, D. D. and Padden, F. J. Macromolecules 1993, 26, 3494. 